Lucrările Seminarului Geografic Dimitrie Cantemir

Vol. 44, October 2017, pp. 101-108

http://dx.doi.org/10.15551//sgdc.v44i0.07

\title{
Á propos de l'ouvrage « le vivant en ville, nouvelles émergences : recherches et publication de synthèse »
}

Irina Rotaru ${ }^{1}$, Bernard Lensel ${ }^{1}$

${ }^{1}$ Métropole de Lyon, Urbanistes des Territoires, France

To cite this article: Rotaru, I., Lensel, B. (2017). Á propos de l'ouvrage « le vivant en ville, nouvelles émergences : recherches et publication de synthèse ». Lucrările Seminarului Geografic Dimitrie Cantemir, Vol. 44, pp. 101-108. DOI: 10.15551/lsgdc.v44i0.07

To link to this article: http://dx.doi.org/10.15551/lsgdc.v44i0.07 


\title{
Á PROPOS DE L'OUVRAGE « LE VIVANT EN VILLE, NOUVELLES EMERGENCES : RECHERCHES ET PUBLICATION DE SYNTHÈSE » 1
}

\author{
Irina Rotaru ${ }^{2}$, Bernard Lensel $^{3}$
}

Résumé. Cette contribution présente de manière synthétique le contenu du livre «Le vivant en ville, nouvelles émergences : recherches et publication de synthèse » paru fin 2016, et les activités menées par la Métropole de Lyon ainsi que par Vétagro-Sup et l'Université de Lyon sur la nature et le bien-être en ville. Elle suit les quatre grandes thématiques abordées par ces recherches : 1 . l'articulation entre gouvernance et vivant en ville ; 2 . les réponses envisagées face aux enjeux de l'intensité urbaine ; 3 . la place et l'enjeu de l'agriculture urbaine ; 4. la question de l'animalité et du bien-être en ville.

Mots-clés : stratégie territoriale - ville durable - écosystème urbain - intensité urbaine agriculture urbaine - futurs urbains - nouvelles émergences - bien commun.

\section{Contexte de la publication}

Ces dernières années, de nombreux articles ${ }^{4}$ ou manifestations ont questionné les rapports entre la nature et la ville. Les collectivités locales dans le monde entier ont commencé se préoccuper de ce thème, en initiant des projets de constructions vertes (comme par exemple le fameux Bosco Verticale projeté par Stefano Boeri à Milan dans le contexte de l'exposition universelle 2015 et réadapté par la suite pour la Chine, qui lui a apporté le renom international) et d'éco-quartiers.

Assumant comme objectif l'étude de la ville en tant qu'espace de vie durable (favorable aux générations présentes, mais aussi à celles futures), la démarche du Vivant en ville $e^{5}$ transforme l'essai avec comme fil rouge l'animalité et l'agriculture urbaine. Cette préoccupation est motivée par l'importance actuelle de l'urbain ainsi que par les changements de paradigme, de culture et de vie, définissant un moment clé dans l'histoire de l'humanité.

L'ouvrage « Le vivant en ville, nouvelles émergences : recherches et publication de synthèse » est basé sur une ample variété de recherches des contributeurs avec différentes

\footnotetext{
${ }^{1}$ Ouvrage, édité et diffusé par la Métropole de Lyon (20 rue du Lac, 69003 Lyon), paru en 2016 et faisant suite à une série de symposiums organisés à Lyon sur la question du « vivant en ville » depuis 2007.

${ }^{2}$ Métropole de Lyon, Urbanistes des Territoires

${ }^{3}$ Métropole de Lyon, Urbanistes des Territoires

${ }^{4}$ Comme par exemple le dossier « Nature(s) en ville » de la revue Métropolitiques, février 2013

${ }^{5}$ Série de symposiums (organisés à Lyon en 2007, 2009 et 2011) et recherches qui ont incité et alimenté la publication du livre susmentionné
} 
expériences et professions leur permettant de voir, apercevoir et interpréter ensemble la ville d'une manière compréhensive, globale.
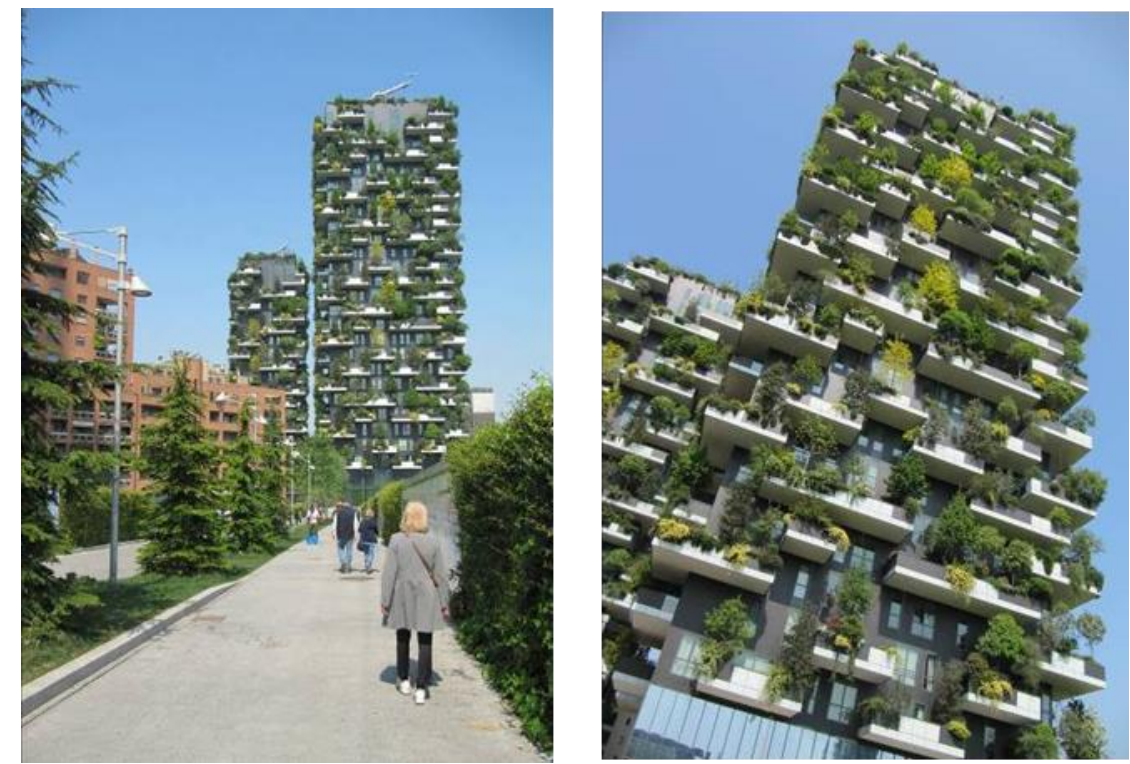

Figure 1 et 2 : Bosco verticale, Milan (Italie) avril 2017

Source: Irina ROTARU

Le premier volet sur l'animalité a été engagé à Lyon depuis 2007, dans le cadre d'un partenariat décisif entre la Métropole de Lyon, l'école VétAgroSup et l'Université de Lyon. Le volet de l'agriculture urbaine est en plein développement actuellement, il interpelle sur les problématiques de santé et de nourriture ; il est traité par le même dispositif partenarial, auquel se sont adjoints le CEREMA ${ }^{6}$ et l'Agence d'Urbanisme de Lyon.

\section{Une démarche globale et cohérente - la gouvernance}

Le modèle fonctionnaliste a généralement expliqué la ville comme ensemble des structures bâties et hommes, tout en ignorant, dans la plupart des cas, les autres éléments vivants, donc la flore et la faune urbaines. Nourri par les fortes tendances actuelles qui accordent plus d'attention aux gens et réorientent les démarches vers des environnements plus humains, la présente synthèse transversale et pluridisciplinaire se concentre sur la grande diversité des composants urbains. Elle intègre plusieurs points de vue et spécialités

6 Le Cerema (Centre d'études et d'expertise sur les risques, l'environnement, la mobilité et l'aménagement) est un établissement public à caractère administratif (EPA), sous la tutelle conjointe du ministère en charge du développement durable et des transports et du ministère en charge de l'urbanisme. Il se distingue comme un centre de ressources et d'expertises techniques et scientifiques en appui aux services de l'État et des collectivités locales, qui repose sur un ancrage territorial fort et sur sa capacité à faire le lien entre les administrations centrales, les services déconcentrés de l'État, les collectivités territoriales et l'ensemble des acteurs qui contribuent à la mise en œuvre des politiques publiques dans les champs de l'aménagement et du développement durable. 
afin de faciliter la compréhension inclusive avec l'ouverture de l'esprit et des perspectives au-delàs des limites arbitraires ou souvent trop rigides des professions. Le but c'est d'informer les stratégies et les modèles futurs pour décrypter et répondre plus efficacement aux besoins réels des collectivités et des territoires.

Sur le plan de la méthodologie, les domaines de l'animalité et de l'agriculture urbaines nécessitent des préalables qui ont été mis en place : d'une part une clarification du jeu des acteurs et de la gouvernance, d'autre part une précision du contexte de l'urbanité, des concepts d'aménagement et de gestion des densités en complémentarité avec les impératifs de drainage du tissu urbain par les trames vertes et bleues ${ }^{7}$, ainsi que par une mobilité multimodale (Lavadinho et Lensel 2012).

Tous ces domaines préfigurent la ville du XXIème siècle, intense et variée à la fois. La relation de l'urbain au rural est un premier jalon, nécessaire à traiter pour ne pas rester dans l'ambiguïté de l'étalement urbain et de la création de secteurs rurbains, consommateurs d'espaces et d'énergie et sociologiquement souvent peu équilibrés.

La végétation comme élément structurant et comme régulateur climatique est un point important pour de nombreuses villes où la lutte contre les épisodes de canicule, la pollution et les inondations, devient partie prenante de stratégies plus larges. L'ambiance que le traitement végétal crée et sa valeur esthétique sont particulièrement importantes pour l'état d'esprit des gens. Bourdeau-Lepage (2012) le précise en mentionnant le besoin de satisfaire «son désir de nature » dans la recherche d'une plus grande qualité de vie, matérielle et immatérielle.

L'agriculture urbaine correspond à une composante croissante du Vivant en ville, qui peut abonder la démarche de circuits courts et de solidarités intra-urbaines ${ }^{8}$, même si elle ne couvrira pas tous les besoins de la consommation des habitants.

Enfin, l'animalité urbaine, vecteur premier du Vivant en ville, représente un indicateur incontournable du bien être en milieu urbain : pour tous les citoyens et notamment pour les

\footnotetext{
${ }^{7}$ La Trame verte et bleue (TVB) est un réseau formé de continuités écologiques terrestres et aquatiques (réservoirs de biodiversité et corridors écologiques) identifiées par les schémas régionaux de cohérence écologique ainsi que par les documents de planification de l'Etat, des collectivités territoriales et de leurs groupements. Les réservoirs de biodiversité sont des espaces dans lesquels celle-ci est la plus riche ou la mieux représentée, où les espèces peuvent effectuer tout ou partie de leur cycle de vie et où les habitats naturels peuvent assurer leur fonctionnement en ayant notamment une taille suffisante. Ceux-ci abritent des noyaux de populations d'espèces à partir desquels les individus se dispersent ou qui sont susceptibles de permettre l'accueil de nouvelles populations. Les corridors écologiques peuvent être linéaires, discontinus ou paysagers et assurent des connexions entre des réservoirs de biodiversité, offrant aux espèces des conditions favorables à leur déplacement et à l'accomplissement de leur cycle de vie. Les TVB contribuent à l'amélioration de l'état de conservation des habitats naturels et des espèces et au bon état écologique des masses d'eau. (Centre de ressources pour la mise en œuvre de la Trame verte et bleue, http://www.trameverteetbleue.fr )

${ }^{8}$ Un circuit court est un mode de commercialisation des produits agricoles qui s'exerce soit par la vente directe du producteur au consommateur, soit par la vente indirecte à condition qu'il n'y ait qu'un seul intermédiaire (définition du Ministère français de l'agriculture). Un Circuit court économique et solidaire (CCES) est une forme d'échange économique valorisant le lien social, la coopération, la transparence et l'équité entre les acteurs de l'échange (Le Labo de l'Economie Sociale et Solidaire www.lelabo-ess.org )
} 
jeunes et les aînés, pour lesquels l'animal peut créer une réelle médiation avec leur environnement pas toujours aussi doux que souhaitable.

Même si longtemps passée sous silence, la nature reste une caractéristique du « fait » métropolitain (Faburel 2015). De manière générale, la ville, les êtres humains et la nature sont les acteurs d'un rapport évolutif. Les nouvelles manières d'intervenir, mais aussi d'analyser et d'interpréter les localités, sont complémentées par des formes d'interaction et de collaboration inédites.

Comme le remarque clairement Frédéric Ségur "L'avenir risque d'imposer une culture des choix différente de celle que l'on connaît aujourd'hui. Au-delà des questions du beau, de l'utile ou du fonctionnel, transparait une autre alternative plus radicale: le dispensable et l'indispensable. La ville-nature doit donc intégrer son héritage historique et esthétique, approfondir sa liaison complexe et sensible avec la société, mais également valoriser sa fonction écologique nécessaire à l'équilibre de fonctionnement d'une ville durable. C'est la condition même de son indispensabilité. »

\section{Passer de la densité à l'intensité urbaine}

Le critère de densité urbaine a pu se justifier dans un contexte coûteux et non structuré d'extension non contrôlée de la ville, qui correspond à l'expression à connotation péjorative d'étalement urbain.

Néanmoins, ce critère est purement quantitatif et il peut en tant que tel être contreproductif, peu adaptable et trop uniformisant.

La ville, lieu de rencontre par excellence, se doit d'être riche et variée au plan des fonctions : c'est ainsi que l'habitat, les services, les commerces sont irrigués par les réseaux de mobilité, aussi multimodaux que possible. Nous avons évoqué les trames vertes et bleues qui peuvent utilement correspondre avec les voies dédiées aux modes doux et actifs (piétons, vélos, trottinettes, ...). L'intensité urbaine synthétise toutes ces démarches et peut être qualifié de densité intelligente et équilibrée (Da Cunha et Kaiser 2009).

Reste à assurer cet équilibre entre les différentes fonctions de la ville, ce qui n'est pas chose facile : la répartition des activités dans la ville, le rapport entre bâti et végétal, les compatibilités et les interactivités entre différents éléments sont autant de variables que le curseur de l'urbanité doit gérer avec doigté.

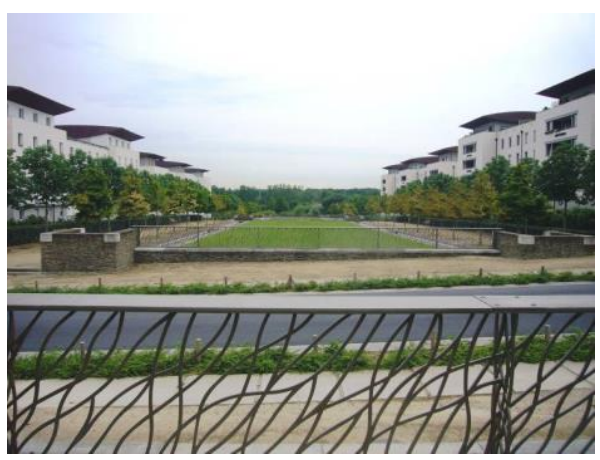

Figure 3 : Nouveau quartier à Carquefou, Nantes Métropole (France) Source : Bernard LENSEL 


\section{L'émergence décisive de l'agriculture en ville et l'intérêt accru pour le végétal}

La pratique de l'agriculture en ville est ancienne; dans des civilisations très différentes, les enceintes de la ville englobaient des activités agricoles et d'élevage, que ce soit chez les Aztèques ${ }^{9}$, dans les entités gallo-romaines ou dans les villes médiévales de l'Europe occidentale (Galarneau et Cousineau Morin 2012), deux raisons militaient pour ce type de disposition: la pratique du circuit court, logique en temps de difficultés à se déplacer, et l'anticipation de conflits et de sièges des villes inhérents à ces conflits.

Les raisons de la nouvelle émergence de l'agriculture tiennent à des raisons partiellement différentes : si la notion de circuit court réapparaît, ce sont surtout les circonstances climatiques et une réponse à la densification parfois trop monocritère qui déterminent ce mouvement. Plus généralement, la montée de l'écologie urbaine tient à une réaction contre l'urbanisme du tout béton et à une recrudescence de l'intérêt pour la nature dont le citoyen de l'urbs se sent coupé, souvent à juste titre. L'action pédagogique vient alors en renfort, avec les fermes en milieu urbain et les jardins liés aux écoles.

On peut distinguer donc deux séries de motivations principales de la végétation en ville, une «alimentaire », verdure comme possibilité de nourrir localement les habitants et l'autre en relation avec la qualité du cadre de vie (image urbaine, air, eau, confort philologique). Autour de cette deuxième catégorie d'orientations, de nombreuses études ont été initiées récemment par les collectivités locales, avec parfois la promotion des outils innovants pilotes pour la gestion durable des projets urbains, comme par exemple la « charte de l'arbre » mise en place depuis 2011 par l'unité « Arbres et paysages » du Grand Lyon. Par la suite, cet outil a été intégré dans un système prospectif plus avancé permettant l'adaptation des projets urbains en fonction du changement climatique (Luce Ponsar).

Selon les expériences analysées par Majorie Musy «Pour le confort d'été dans les bâtiments, les enveloppes végétales, qui participent également au confort dans les rues, sont intéressantes dans le cas des bâtiments peu isolés. En ce qui concerne la limitation du ruissellement, les noues sont les plus performantes. Il apparaît donc que l'urbaniste, le paysagiste, l'architecte et le gestionnaire des espaces verts ont les clés pour concevoir des aménagements qui permettent de répondre aux différents enjeux (ceux de climat, de hydrologie, de l'énergie, de l'acoustique et des ambiances traités ici, auxquels s'ajoutent d'autres enjeux comme la biodiversité, le bilan carbone, les relations sociales) »

Par ailleurs, comme illustré par Valérie Demers, Mikael Saint-Pierre \& Maxime Larose, la végétation peut représenter un instrument très puissant de réintégration des friches urbaines dans la vie et dans les circuits des villes, tout en favorisant le développement « d'un sentiment collectif de pouvoir agir sur son territoire ».

\footnotetext{
${ }^{9}$ Qui ont contribué au développement des adaptations innovantes afin d'éliminer les contraintes courantes des activités agricoles liées au caractère urbain des espaces, comme par exemple l'aquaponie (procédé agricole où les plantes ne poussent sur un substrat neutre - sable ou roches par exemple - régulièrement irrigué éliminant ainsi la nécessité de disposer des surfaces assez importantes avec un sol fertile et diminuant la quantité d'eau consommée).
} 


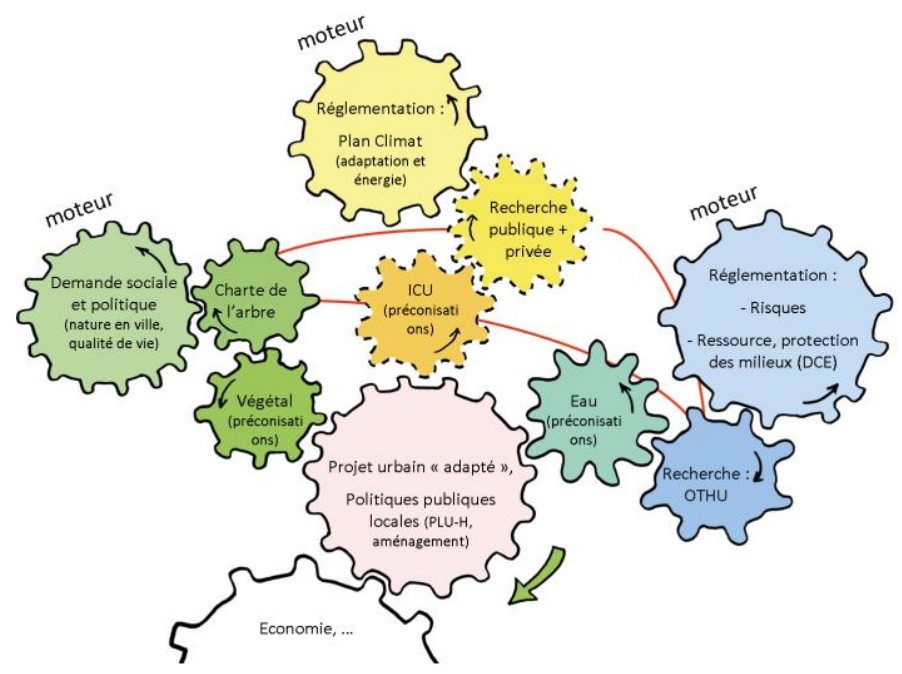

Figure 4: Les moteurs (réglementation, demande sociale) nécessitant des « organes de transmission $»$ (recherche locale, partenariats avec les professionnels) pour faire avancer le projet urbain vers la prise en compte des enjeux de l'adaptation au changement climatique.

Source : Communauté Urbaine du Grand Lyon, 2014, cité par Luce Ponsar dans sa contribution.

\section{L’animalité urbaine comme fil conducteur}

L'une des composantes du vivant en ville concerne l'animalité et cette démarche a une antériorité sur les autres volets ; en effet, la reconnaissance du bien être en ville concerne tout autant l'animal que l'homme au sens générique du terme.

L'entente entre les êtres vivants, dans la mesure évidemment de leur compatibilité de modes de vie, peut donc être un critère primordial de qualité urbaine. La biodiversité est à la clé un indicateur de la qualité environnementale, notamment en milieu urbain.

L'animalité apparaît aussi comme une médiation de certains problèmes humains, particulièrement chez les juniors et chez les seniors, ainsi qu'en milieu hospitalier. En plus, certains oiseaux présents dans l'espace urbain servent de composteurs de déchets ménagers.

Par ailleurs, le lien entre animalité et végétal est très prégnant, comme le montre de façon évidente l'action des abeilles, qui est incontournable pour certaines plantes, dont elle assure la pérennité. En partant du fonctionnement naturel de ces écosystèmes, la société berlinoise ECF (Efficient City Farming) ${ }^{10}$ fondée en 2012 a mis au point une "ferme-container", prototype d'une agriculture urbaine qui allie pisciculture et cultures maraîchères. Il s'agit d'un container qui abrite l'élevage de poissons, coiffé d'une serre où poussent des légumes. Les bactéries transforment l'ammonium des déjections des poissons en nitrates, qui servent d'engrais pour les cultures un étage au-dessus.

\footnotetext{
${ }^{10} \mathrm{http} / / /$ www.ecf-farmsystems.com/en/
} 
Selon François Darribehaude \& Sébastien Gardon «Pour préparer la Ville de demain, il apparaît indispensable de définir la place, le rôle et le statut de ces occupants, parmi lesquels les animaux tout comme d'ailleurs les végétaux. »

\section{Une interaction des différents volets du Vivant en ville pour le XXIème siècle}

Les nouvelles émergences en termes de Vivant en ville sont manifestement complémentaires et devraient se comporter de façon interactive : une réelle gouvernance est à confirmer en vue d'un jeu des acteurs dès l'amont des processus et s'inscrivant sur le long terme. La prise en compte équilibrée des trois volets du développement soutenable/durable est un passage nécessaire pour l'élaboration des projets et de la gestion urbaine.

Ces émergences se poursuivent et offrent un caractère très prometteur pour l'élaboration de la ville du XXIème siècle : un comparatif des expériences dans d'autres pays, même lointains, est et sera très intéressant ; la protection physique et juridique de l'animal (et pourquoi pas du végétal) va se renforcer, à juste titre; le développement de l'agriculture urbaine, avec de nouvelles pratiques comme les ruches et le pastoralisme urbains; les trames mixtes en ville, en référence avec les expériences espagnoles ${ }^{11}$ d'alternance d'espaces circulés et d'espaces verts dédiés aux modes doux et actifs de déplacement.

Au-delà des préoccupations écologiques stricto sensu, protéger les autres êtres vivants devient finalement une condition indispensable (mais, évidemment, pas suffisante) pour maintenir un cadre de vie favorable aux hommes, pour assurer leur bien-être ; approche vue par les responsables de la Métropole de Lyon comme part d'une «façon nouvelle et durable de construire et d'habiter la ville». Ayant une forte influence sur les expériences sociales et sur l'expérimentation des villes, la nature (flore et faune) peut être facilement interprétée comme un bien commun ${ }^{12}$, de la gestion duquel dépend le devenir des sociétés et de l'urbain. Par ailleurs, dans certains contextes, elle peut apparaître de plus en plus souvent comme un facteur de distinction des espaces urbains et des quartiers, mais aussi de discrimination et parfois de ségrégation sociale. Dans ce sens, les Plans Climat ${ }^{13}$ présentent une opportunité pour être plus à l'écoute des habitants et des personnes vulnérables en intégrant les enjeux de santé publique (ombrage des voies cyclables, «oasis de fraîcheur » etc.).

Analysant les différentes connotations et rôles de la nature en ville, Guillaume Faburel étudie le vivant comme puissance d'agir des habitants, en connexion avec concepts

\footnotetext{
${ }^{11}$ les expériences espagnoles mentionnées concernent notamment les villes de Barcelone (Catalogne) et de Vitoria-Gasteiz (Pays Basque) ; elles sont dénommées "Supermanzanas" de façon imagée et correspondent à des îlots urbains caractérisés par une alternance de trames circulées et de trames modes doux, vertes et bleues.

${ }^{12}$ Selon François FLAHAULT (2013), le bien commun est ce qui est profitable à long terme pour l'ensemble des membres de la société. http://www.participation-et-democratie.fr/es/dico/biencommun

${ }^{13}$ Conçu au niveau national ou régional ou local, le Plan Climat est un outil stratégique pensé pour contrecarrer le changement climatique. Il encourage les gestes citoyens par la sensibilisation et l'information ; favorise les sources d'énergie non émettrices de carbone et insère l'écologie dans l'économie grâce à des mécanismes novateurs, efficaces et flexibles.
} 
tels que « expertise d'usage », « savoirs d'usage » et « maîtrise d'usage » et le désir d'habiter autrement traduit par une évolution écologique des modes de vie observée par Dobré et Juan (2009). Laurène Wiesztort signale l'apparition en ville de nouvelles formes de nature urbaine, qu'elle nomme «nature filtrée ${ }^{14}$.

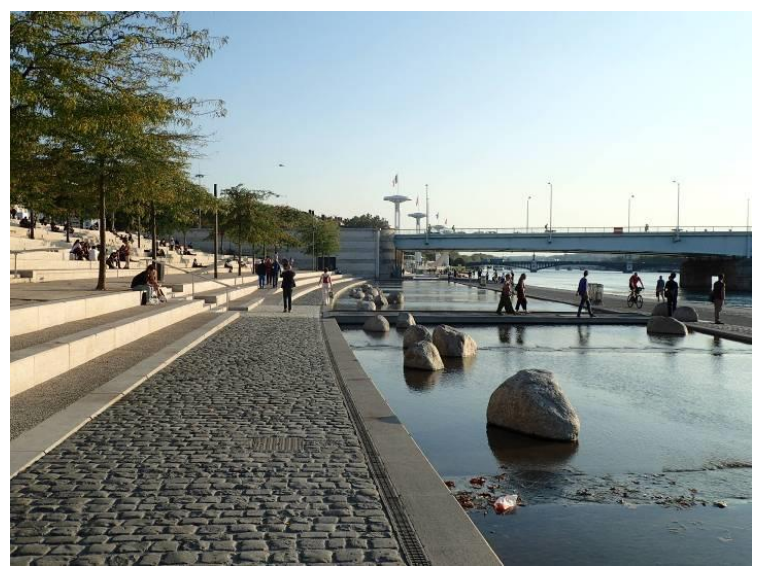

Figure 5 : Aménagement des Berges du Rhône, à Lyon (France)

Source : Bernard LENSEL

Cet effort collectif qui a abouti dans la publication du livre Le vivant en ville, nouvelles émergences a réussi à croiser la recherche publique avec celle privée et les initiatives empiriques en termes d'aménagement (Luce Ponsar, Chargée de mission Plan Climat, Métropole de Lyon). Il a accrédité et encouragé ainsi nouveaux rapports, plus égalitaires, entre les différents partenaires impliqués et a contribué à l'amélioration du lien entre chercheurs, praticiens et société civile en privilégiant une organisation guidée par l'efficacité. Par ailleurs, il a conduit à la consolidation d'un espace de réflexion et de prospective ouvert, interdisciplinaire et global, répondant aux enjeux, aux exigences et aux tendances contemporaines.

\section{Références bibliographiques}

1. Bourdeau-Lepage L., 2012. Regards sur la ville, Paris, Economica (Anthropos-géographie).

2. Dobré M., Juan S. (sous la dir.) (2009), Consommer autrement - La réforme écologique des modes de vie, l'Harmattan, Paris, $312 \mathrm{p}$

3. Da Cunha, A., Kaiser, C., 2009. "Densité, centralité et qualité urbaine : la notion d'intensité, outil pour une gestion adaptative des formes urbaines? », Revue Urbia ${ }^{\circ} 9$, Lausanne

4. Faburel G., 2015. «Construction démocratique de l'agir métropolitain. Métropole 1 - Habitants 0 . Vers un match retour?», L'esprit des villes, ${ }^{\circ} 2,155-176$, Infolio.

5. Galarneau V. et Cousineau Morin P., 2012. L'agriculture urbaine, composante essentielle des collectivités viables, mémoire coordonné par Christian Savard et Jeanne Robin et présenté par Vivre en Ville à L'Office de consultation publique de Montréal dans le cadre de la consultation publique sur l'agriculture urbaine Consultable en ligne à https://vivreenville.org/media/4480/venv_agriurb_collviables_mtl_14juin2012.pdf

6. Lavadinho S. et Lensel B., 2012. Mobilités vertes et actives, Revue Collage, Zürich

${ }^{14}$ Résultant partiellement de la volonté de créer des continuums végétaux 